\title{
Investigation of the Effect of Pulsatile and Nonpulsatile Flow on Kidney in Coronary Surgery With NIRS
}

\author{
Ferhat Borulu, ${ }^{1}$ Muhammet Onur Hanedan, ${ }^{2}$ Ceyhun Coşkun, ${ }^{3}$ İzzet Emir, ${ }^{4}$ Ilker Mataraci ${ }^{2}$ \\ ${ }^{1}$ Department of Cardiovascular Surgery, Atatürk University Faculty of Medicine, Erzurum, Turkey; ${ }^{2}$ Department of Cardiovascular \\ Surgery, University of Health Sciences Ahi Evren Education and Researches Hospital, Trabzon, Turkey; ${ }^{3}$ Department of \\ Cardiovascular Surgery, Yalvaç Public Hospital, Isparta, Turkey; ${ }^{4}$ Department of Cardiovascular Surgery, Erzincan University, Faculty \\ of Medicine, Erzincan, Turkey
}

\section{ABSTRACT}

Background: Acute renal insufficiency is a significant cause of morbidity and mortality after coronary artery bypass grafting performed with cardiopulmonary bypass. Functional near-infrared spectroscopy (fNIRS) is an emerging brain-imaging technique that can be used to detect organ perfusions in adults. This study aims to determine the effects of pulsatile and nonpulsatile flow on renal circulation by using functional near-infrared spectroscopy and biochemical markers.

Methods: Forty patients, who had undergone isolated CABG between March 2014 and July 2014 in the authors' clinic, were included in the study. Patients were divided in the pulsatile and nonpulsatile groups by simple randomization.

Results: Urine outputs statistically were higher in the pulsatile group, during CPB $(P=.045)$. Renal perfusion measurements via fNIRS intra-operatively statistically were parallel between the groups. When we compared biochemical markers within the groups, in the nonpulsatile group, creatinin statistically is higher on the postoperative first day $(P=.003)$, and BUN statistically is higher on the postoperative first $(P=.015)$, second $(P=.001)$, and fifth $(P=.020)$ days, according to preoperative value. In the pulsatile group, only the postoperative second day BUN is higher $(P=.007)$.

Conclusion: fNIRS is being used to track cerebral functions. During operation, it also provides a correct observation for blood feeding of somatic organs, such as the kidneys, but it requires more clinical study to be accepted as routine.

\section{INTRODUCTION}

Recent studies have shown that cardiac surgery with cardiopulmonary bypass (CPB) induces a systemic inflammatory response syndrome (SIRS) [Paparella 2002]. Postoperative complications like myocardial dysfunction,

Received December 3, 2019; accepted March 30, 2020.

Correspondence: Ferbat Borulu, Department of Cardiovascular Surgery, Atatürk University Faculty of Medicine, Erzurum, Turkey (e-mail: fborulu@gmail.com). respiratory failure, renal and neurologic dysfunction, bleeding disorders, altered liver function, and ultimately, multiple organ failure (MOF) may develop by this inflammatory reaction. Even with advances in methods and technologies, postoperative acute renal insufficiency continues to be a significant cause of morbidity and mortality after coronary artery bypass grafting (CABG) performed with CPB [Sahin 2015].

Functional near-infrared spectroscopy (fNIRS), which can assess the relative changes of oxy hemoglobin (HbO), deoxy hemoglobin $(\mathrm{Hb})$, and total hemoglobin $(\mathrm{HbT})$ in the cortical regions of the brain, is an emerging brain-imaging technique [Sai 2014]. In the pediatric population, fNIRS also is used to detect organ perfusions like kidneys [Ricci 2015]. In a limited number of studies, fNIRS is presented to detect organ perfusions in adults [Toraman 2012].

In this prospective study, we aimed to assay the effects of pulsatile and nonpulsatile flow on renal circulation, during $\mathrm{CPB}$ in $\mathrm{CABG}$ patients by using functional near-infrared spectroscopy and biochemical markers.

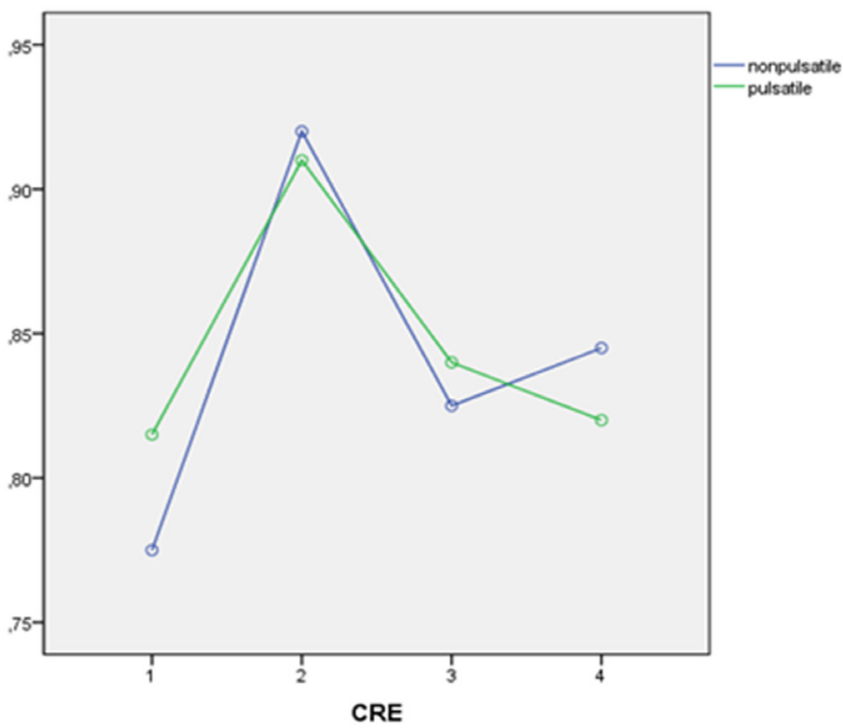

Figure 1. Creatinine measures between the different flow patterns $(P=.953)$. 


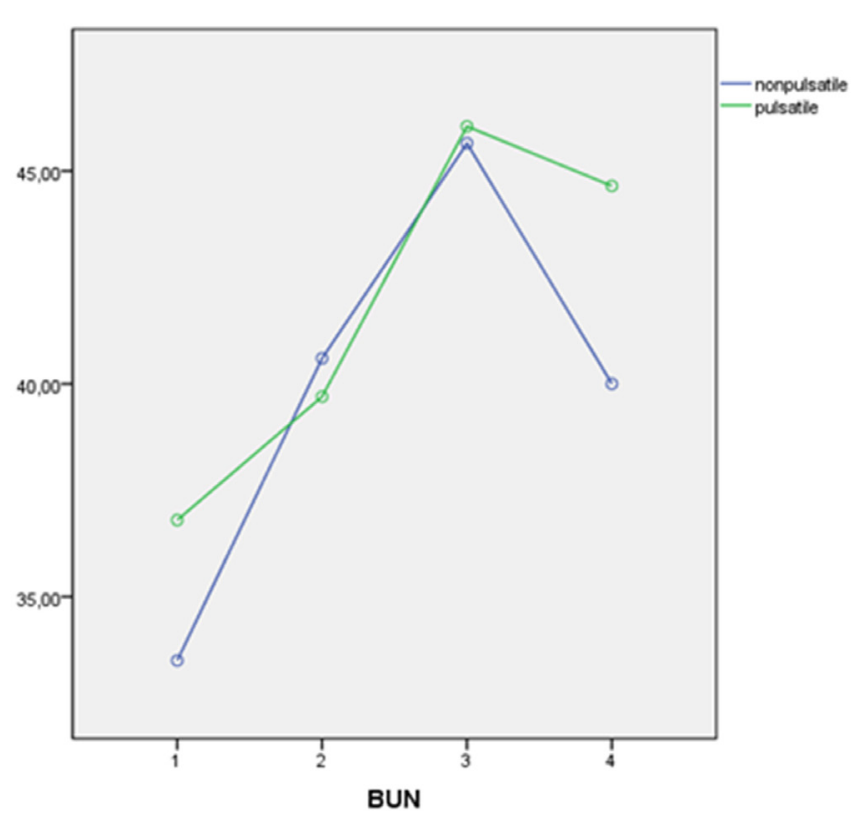

Figure 2. BUN measures between the different flow patterns $(P=.601)$.

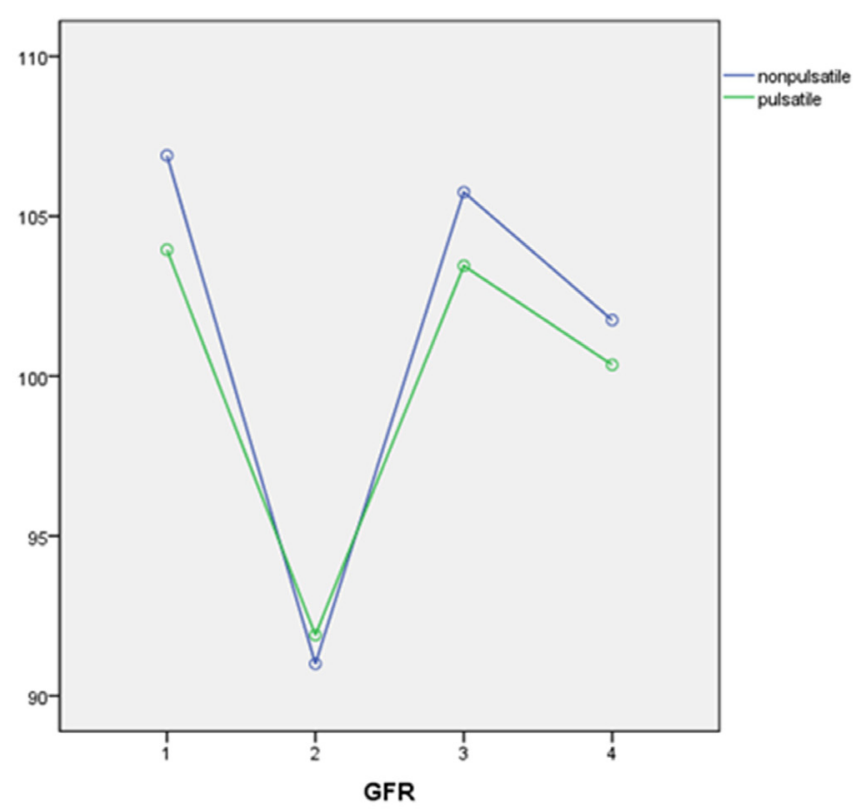

Figure 3. GFR measures between the different flow patterns $(P=.875)$.

\section{PATIENTS AND METHODS}

\section{Study group}

Forty patients, who had undergone isolated CABG between March 2014 and July 2014 in the authors' clinic, were included in the study. Patients were randomized into two groups by flipping a coin.

All patients were operated on by one expert surgeon. Patients with renal artery stenosis, renal failure, low ejection
Table 1. Preoperative demographics, clinical and echocardiographic findings of the patients

\begin{tabular}{lccc}
\hline & Pulsatile $(\mathrm{N}=20)$ & Nonpulsatile $(\mathrm{N}=20)$ & $P$ \\
\hline Gender & & & \\
Male & $17(85 \%)$ & $17(85 \%)$ & 1.000 \\
Female & $3(15 \%)$ & $3(15 \%)$ & 1.000 \\
Age & $60.45 \pm 7.79$ & $57.40 \pm 7.59$ & .217 \\
Hypertension & $12(60 \%)$ & $14(70 \%)$ & .507 \\
Diabetes mellitus & $3(15 \%)$ & $5(25 \%)$ & .695 \\
KOAH & - & $2(10 \%)$ & .487 \\
Cigarette & $15(75 \%)$ & $12(60 \%)$ & .311 \\
Alcohol & $2(10 \%)$ & $1(5 \%)$ & 1.000 \\
Hyperlipidemia & $15(75 \%)$ & $14(70 \%)$ & .723 \\
SVO & - & $1(5 \%)$ & 1.000 \\
BMl (body mass index) & $25.20 \pm 2.80$ & $26.32 \pm 2.01$ & .155 \\
EF & $60.5 \pm 5.59$ & $59.5 \pm 5.10$ & .558
\end{tabular}

Table 2. Operative variables

\begin{tabular}{lccc}
\hline & Pulsatile & Nonpulsatile & $P$ \\
\hline Perfusion current $(\mathrm{mL} / \mathrm{min})$ & $4070 \pm 388.79$ & $4185 \pm 277.72$ & .289 \\
Perfusion time $(\mathrm{min})$ & $88.10 \pm 30.19$ & $83.20 \pm 13.18$ & .512 \\
Aorta clamp time $(\mathrm{min})$ & $54.50 \pm 18.52$ & $57.30 \pm 11.40$ & .569 \\
Blood pressure $(\mathrm{mmHg})$ & $68.10 \pm 7.43$ & $67.75 \pm 7.32$ & .882 \\
CPB urine output $(\mathrm{mL})$ & $567.50 \pm 195.6$ & $460 \pm 120.961$ & .045
\end{tabular}

fraction (EF), and emergency status were not included in the study. All patients gave written consent for the study and approval of the study was obtained from the hospital's ethics committee.

\section{NIRS}

Renal doppler ultrasound (Mindray 7-convex probe) imaging preoperatively was performed to detect the optimal positioning of the NIRS probe and renal artery disease. The closer part of the skin to renal parenchyma was marked and the distance between them measured. INVOS 5100C Cerebral/Somatik oximetry probes were placed to the marked area. Peripheral oxygen saturation, blood pressure, and renal parenchyma oxygenation were measured before anesthetic induction, before intubation, initiating to $\mathrm{CPB}$, fifth minute of the $\mathrm{CPB}$, tenth minute of the cross clamping, twentieth minute of the cross clamping, end of the $\mathrm{CPB}$, and end of the operation.

\section{Surgical technique}

Under general anesthesia and orotracheal intubation, all patients underwent CABG after full sternotomy. After going on $\mathrm{CPB}$ with moderate hypothermia (nasopharyngeal 
Table 3. fNIRS, blood pressure and peripheral saturation measurements during operation

\begin{tabular}{|c|c|c|c|c|}
\hline \multirow[t]{2}{*}{ Pre-induction } & Right/Left & $80.60 \pm 4.94 / 80.85 \pm 3.32$ & $81.40 \pm 6.37 / 80.10 \pm 5.22$ & $.66 / .59$ \\
\hline & PerSO2 & $96.30 \pm 0.47$ & $95.95 \pm 1.43$ & .31 \\
\hline \multirow[t]{2}{*}{ Post-intubation } & Right/Left & $82.95 / 83.35 \pm 5.79 / 4.93$ & $82.85 / 81.60 \pm 7.05 / 5.59$ & $.96 / .30$ \\
\hline & Peripheral & $99.80 \pm 0.41$ & $99.45 \pm 0.60$ & $.04 *$ \\
\hline \multirow[t]{3}{*}{ CPB entry } & Right/Left & $76.45 / 76.80 \pm 5.12 / 4.43$ & $78.70 / 77.90 \pm 6.54 / 5.79$ & $.23 / .50$ \\
\hline & TA & $62.35 \pm 13.59$ & $58.30 \pm 12.07$ & .32 \\
\hline & Peripheral & $99.35 \pm 1.04$ & $97.90 \pm 4.86$ & .20 \\
\hline CPB 5th minute & Right/Left & $75.15 / 75.60 \pm 5.64 / 5.17$ & $77.90 / 76.55 \pm 6.66 / 6.30$ & $.16 / .60$ \\
\hline \multirow{2}{*}{ Cross clamp 10th minute } & TA & $63.90 \pm 11.76$ & $60.45 \pm 9.47$ & .31 \\
\hline & Peripheral & $99.35 \pm 0.98$ & $98.80 \pm 1.93$ & .26 \\
\hline \multirow[t]{3}{*}{ Cross clamp 20th minute } & Right/Left & $75.65 / 76.40 \pm 4.99 / 5.49$ & $78.85 / 77.85 \pm 5.42 / 5.18$ & $.06 / .39$ \\
\hline & TA & $65.10 \pm 9.17$ & $64.05 \pm 10.40$ & .73 \\
\hline & Peripheral & $99.00 \pm 1.74$ & $98.30 \pm 2.08$ & .25 \\
\hline \multirow[t]{3}{*}{ When removing cross clamp } & Right/Left & $74.65 / 76.20 \pm 6.01 / 6.02$ & $78.15 / 76.90 \pm 6.93 / 6.92$ & $.09 / .73$ \\
\hline & TA & $61.30 \pm 9.49$ & $56.15 \pm 14.70$ & .19 \\
\hline & Peripheral & $99.40 \pm 0.88$ & $98.10 \pm 2.61$ & $.04 *$ \\
\hline CPB exit & Right/Left & $76.70 / 78.10 \pm 5.91 / 7.00$ & $78.40 / 78.05 \pm 6.16 / 5.88$ & $.37 / .98$ \\
\hline
\end{tabular}

temperature, 30 to $32^{\circ} \mathrm{C}$ ), (flow rate: $2.5 \mathrm{~L} / \mathrm{min} / \mathrm{m}^{2}$ ), mean systemic pressure was maintained at $60 \mathrm{mmHg}$. Myocardial protection was achieved by antegrade administration of blood cardioplegic solution on induction and continued with administration of cold-blood cardioplegic doses every 20 minutes, in accordance with our institution's routine. A final warm-blood dose was administered before releasing the cross clamp.

For half of the patients, pump was operated in pulsatile mode, while for others it was operated in nonpulsatile mode. The pump that was operated in nonpulsatile mode before placing the cross clamp in the pulsatile mode group was changed to pulsatile mode with placing the cross clamp. CPB was established with a standard 6 " roller pump using the Terumo ${ }^{\circledR}$ Advanced Perfusion System 1 (Terumo Cardiovascular Group, Ann Arbor, MI) heart-lung machine. The extracorporeal circuits consisted of biocompatible custom heparinalbumin coated circuits (Bioline®, Maquet Cardiopulmonary
AG., Hirrlingen, Germany) and the membrane oxygenator was Quadrox-I Adult (Sofline® Maquet Cardiopulmonary AG., Hirrlingen, Germany). Systemic perfusion was converted to pulsatile flow at a rate of 70 beats per minute for all patients randomized into this group under cross clamp. Pulsatile flow was generated with the following set parameters: base $60 \%$ and width $60 \%$. In contrast, the nonpulsatile group received continuous flow throughout the procedure.

\section{Markers}

For both patient groups, in the preoperative period and on the first, second, and fifth postoperative days, patients were checked for kidney and liver functions and values of hemogram, troponin, and glucose were recorded.

Statistical analysis: Statistical evaluation was done with SPSS v16.0 (SPSS Inc., Chicago, IL) packaged software. The results were reported as mean \pm standard $(\mathrm{SD})$ for quantitative variables and percentages for categorical variables. Pearson 
Table 4. Comparison of hemogramand biochemical data between groups

\begin{tabular}{|c|c|c|c|c|}
\hline \multirow[t]{3}{*}{ WBC } & Preop & $7.91 \pm 2.25$ & $8.02 \pm 2.38$ & .882 \\
\hline & 2nd day & $11.97 \pm 2.88$ & $14.01 \pm 3.78$ & .063 \\
\hline & 5 th day & $8.19 \pm 2.20$ & $9.91 \pm 3.29$ & .060 \\
\hline \multirow{3}{*}{ HGB } & 1st day & $10.13 \pm 1.31$ & $9.37 \pm 0.85$ & $.035 *$ \\
\hline & 2nd day & $9.03 \pm 0.73$ & $8.29 \pm 0.72$ & $.003 *$ \\
\hline & 5th day & $8.57 \pm 0.67$ & $8.63 \pm 0.77$ & .779 \\
\hline \multirow[t]{2}{*}{$\mathrm{HCT}$} & Preop & $41.18 \pm 3.32$ & $42.27 \pm 4.99$ & .424 \\
\hline & 1st day & $30.30 \pm 4.08$ & $28.21 \pm 2.53$ & .059 \\
\hline \multirow{3}{*}{ PLT } & 1 st day & $160.05 \pm 39.90$ & $175.80 \pm 40.85$ & .225 \\
\hline & 2nd day & $152.70 \pm 37.43$ & $173.95 \pm 44.85$ & .112 \\
\hline & 5th day & $201.95 \pm 48.37$ & $233.50 \pm 68.64$ & .101 \\
\hline \multirow[t]{4}{*}{ BUN } & Preop & $37.10 \pm 9.97$ & $33.50 \pm 7.96$ & .215 \\
\hline & 1st day & $39.70 \pm 11.20$ & $40.60 \pm 13.93$ & .823 \\
\hline & 2nd day & $46.05 \pm 16.72$ & $45.65 \pm 14.99$ & .937 \\
\hline & 5th day & $44.65 \pm 17.73$ & $40.00 \pm 12.90$ & .349 \\
\hline \multirow[t]{2}{*}{ CREATINE } & Preop & $0.81 \pm 0.24$ & $0.77 \pm 0.20$ & .600 \\
\hline & 1 st day & $0.91 \pm 0.34$ & $0.92 \pm 0.30$ & .915 \\
\hline
\end{tabular}

$\chi^{2}$ test (or the Fisher exact test) was used for comparing categorical variables, and independent samples t-test and paired t test were used to compare continuous variables. Repeated measures analysis of variance was used to analyze biochemical markers within the groups. A $P$-value of $<.05$ was considered to be statistically significant.

\section{RESULTS}

The mean age was $60.45 \pm 7.79$ years in the pulsatile group and $57.40 \pm 7.59$ years in the nonpulsatile group. Three patients $(15 \%)$ were women in both groups. New Euroscore II was calculated $0.94 \pm 0.40$ and $0.86 \pm 0.35$, respectively, in the pulsatile and nonpulsatile groups $(P=.507)$. Body mass index (BMI) and EF were similar in both groups, and $P$ values were 0.155 and 0.558 , respectively. Patient preoperative data is summarized in Table 1. Kidney to skin measurements preoperatively were recorded and statistically were similar in both groups.

The mean perfusion flow was $4070 \pm 388.79 \mathrm{~mL} / \mathrm{minute}$ for the pulsatile group and $4185 \pm 277.72 \mathrm{~mL} / \mathrm{minute}$ for the nonpulsatile group $(P=.289)$. Mean blood pressure during $\mathrm{CPB}$ was $68.10 \pm 7.43$ and $67.75 \pm 7.32$, respectively, in the pulsatile and nonpulsatile groups $(P=.882)$. Urine output statistically was higher in the pulsatile group, during $\mathrm{CPB}$ $(P=.045)$ (Table 2).

Renal perfusion measurements via fNIRS intra-operatively were statistically parallel between the groups (Table 3 ).

When complete blood count (CBC) parameters were analyzed, white blood cell (WBC) count on the postoperative first day $(P=.008)$, hemoglobin values on the postoperative first $(P=.035)$ and second days $(P=.003)$, and hematocrit rates on the postoperative second day $(P=.003)$ were statistically different in favor of the pulsatile group. There was no difference between the groups when we compare biochemical markers (Table 4).

However, when we compare biochemical markers within the groups, in the nonpulsatile group, creatinin statistically is higher on the postoperative first day $(P=.003)$ and BUN statistically is higher on the postoperative first $(P=.015)$, second $(P=.001)$, and fifth $(P=.020)$ days, according to preoperative value. In the pulsatile group, only postoperative second day BUN was higher, compared with preoperative numbers $(P=.007)$ (Table 5). 
Table 5. Biochemical markers within groups

\begin{tabular}{lccc}
\hline & & Mean \pm SD & $P$ \\
\hline Nonpulsatile BUN & Preop & $33.50 \pm 1.78$ & \\
& Postop 1st day & & $.015^{*}$ \\
& Postop 2nd day & $45.65 \pm 3.55$ & $.001^{*}$ \\
& Postop 5th day & $40.00 \pm 2.88$ & $.020^{*}$ \\
Nonpulsatile creatine & Preop & $0.77 \pm 0.45$ & \\
& Postop 1st day & & $.003^{*}$ \\
& Postop 2nd day & $0.82 \pm 0.69$ & .141 \\
& Postop 5th day & $0.84 \pm 0.66$ & .092 \\
Pulsatile BUN & Preop & $37.10 \pm 9.97$ & \\
& Postop 1st day & & .175 \\
& Postop 2nd day & $46.05 \pm 16.72$ & $.007^{*}$ \\
& Postop 5th day & $44.65 \pm 17.73$ & .083 \\
Pulsatile creatine & Preop & $0.81 \pm 0.24$ & \\
& Postop 1st day & & .114 \\
& Postop 2nd day & $0.84 \pm 0.33$ & .624 \\
& Postop 5th day & $0.82 \pm 0.25$ & .909 \\
& & &
\end{tabular}

\section{DISCUSSION}

Coronary artery disease is strongly associated with chronic renal failure and changes in renal arteries [Fuiano 2005]. Patients referred for coronary artery bypass grafting are older, have poorer left ventricular function, and lower GFR because of the increased use of angioplasti [Grover 2001]. Preoperatively, GFR is the most powerful predictor of postoperative acute renal failure. $\mathrm{CPB}$ also is an independent predictor of renal failure after CABG [Onorati 2007].

Renal dysfunctions can occur during $\mathrm{CPB}$, due to exposure of blood to a foreign surface, hemodilution, hypothermia, and nonpulsatile blood flow. Renin secretion, during nonpulsatile flow, may affect the kidneys more than other organs [Sievert 2012]. Sympathetic activation with nonpulsatile blood flow results in vasoconstriction and increased afterload. This may happen as a result of not stimulating the arterial baroreceptors to a similar degree as pulsatile devices or normal controls [Markham 2013].

Changes in kidneys or other organs give symptoms no sooner than 24 hours, and because of this detecting injuries in kidney early has become more important [Toraman 2012]. Diaz et al found that early detection of renal or hepatic injury improves the results [Diaz 2008]. In a study from the Duke University database on 2,672 patients undergoing elective CABG with $\mathrm{CPB}$, acute kidney injury requiring renal replacement therapy occurred in only $0.7 \%$ of patients, but was associated with $28 \%$ mortality, compared with $1 \%$ in patients without kidney injury [Conlon 1999]. Mortality rates increase to $88 \%$ in patients with acute renal failure after cardiac surgery [Khilji 2004].

Increasing evidence supports a shift from nonpulsatility to pulsatility in open-heart surgeries, with clinical evidence showing better cardiac, renal and pulmonary outcomes in patients receiving pulsatile perfusion [Bayram 2012]. Benefits of pulsatile flow include reduction of the systemic inflammatory response syndrome associated with bypass, decreased need for inotropic support, shortened hospital stay, and superior organ preservation [Mohammadzadeh 2013]. Our study also demonstrated improved biochemical and hemogram findings, and urine output in the pulsatile group.

The literature often states that postoperative somatic organ damage is a factor increasing cardiac surgical mortality and morbidity. Hence, there is a need for organ-protective strategies, and new tracking modalities may help with diagnosing somatic organ dysfunctions earlier [Toraman 2012]. In our study, we used fNIRS to track renal function during operations. We found that fNIRS measurements were equal for both groups; similarly, we did not observe any renalrelated complications during the postoperative period in both groups.

\section{CONCLUSION}

Presently, fNIRS is used in tracking cerebral functions during operations. It also provides accurate observation of blood feeding for somatic organs, such as the kidneys, but further clinical studies are required to justify routine usage. Due to high mortality in $\mathrm{CABG}$ patients having acute renal failure, fNIRS must be considered for operations with pulsatile current in high-risk groups.

\section{REFERENCES}

Bayram H, Erer D, Iriz E, HakanZor M, Gulbahar O, Ozdogan ME. 2012. Comparison of the effects of pulsatile cardiopulmonary bypass, nonpulsatile cardiopulmonary bypass and off-pump coronary artery bypass grafting on the respiratory system and serum carbonyl. Perfusion. 27:378-85.

Conlon PJ, Stafford-Smith M, White WD, et al. 1999. Acute renal failure following cardiac

surgery. Nephrol Dial Transplant 14:1158-62.

Diaz GC, Moitra V, Sladen RN. 2008. Hepatic and renal protection during cardiac surgery. Anesthesiol Clin. 26:565-90.

Fuiano G, Mancuso D, Indolfi C, et al. 2005. Early detection of progressive renal dysfunction in patients with coronary artery disease. Kidney Int 68:2773-2780.

Grover FL, Shroyer AL, Hammermeister K, et al. 2001. A decade's experience with quality improvement in cardiac surgery using the Veterans Affairs and Society of Thoracic Surgeons national databases. Ann Surg 234:464-472; discussion, 472-464.

Khilji SA, Khan AH. 2004. Acute renal failure after cardiopulmonary bypass surgery. J Ayub Med Coll Abbottabad. 16:25-8.

Markham DW, Fu Q, Palmer MD, Drazner MH, Meyer DM, Bethea BT, Hastings JL, Fujimoto N, Shibata S, Levine BD. 2013. Sympathetic neural and hemodynamic responses to upright tilt in patients with pulsatile and nonpulsatile left ventricular assist devices. Circ Heart Fail. Mar;6(2):293-9. 
Mohammadzadeh A, Jafari N, Hasanpour M, Sahandifar S, Ghafari M, Alaei V. 2013. Effects of pulsatile perfusion during cardiopulmonary bypass on biochemical markers and kidney function in patients undergoing cardiac surgeries. Am J Cardiovasc Dis Aug 16;3(3):158-62.

Onorati F, Presta P, Fuiano G, Mastroroberto P, Comi N, Pezzo F, Tozzo C, Renzulli A. 2007. A randomized trial of pulsatile perfusion using an intra-aortic balloon pump versus nonpulsatile perfusion on short-term changes in kidney function during cardiopulmonary bypass during myocardial reperfusion. Am J Kidney Dis Aug;50(2):229-38.

Paparella D, Yau TM, Young E. 2002. Cardiopulmonary bypass induced inflammation: pathophysiology and treatment. An update. Eur J Cardiothorac Surg Feb;21(2):232-44.

Ricci Z, Haiberger R, Tofani L, Romagnoli S, Favia I, Cogo P. 2015. Multisite Near Infrared Spectroscopy During Cardiopulmonary Bypass in Pediatric Patients. Artif Organs. Jul;39(7):584-90.

Sahin V, Akpinar MB, Sevim E, Uyar IS, Abacilar AF, Uc H, Tetik F, Damar E, Okur FF, Alayunt EA. 2015. Preoperative CRP levels is not predictive early renal dysfunction after coronary artery bypass surgery. Int J Clin Exp Med Mar 15;8(3):4146-51.

Sai L, Zhou X, Ding XP, Fu G, Sang B. 2014. Detecting concealed information using functional near-infrared spectroscopy, Brain Topogr. 27(5), 652-662.

Sievert A, Sistino J. 2012. A meta-analysis of renal benefits to pulsatile perfusion in cardiac surgery. J Extra Corpor Technol Mar;44(1):10-4.

Toraman F, Özgen SU, Arıtürk C, Julide S, Erkek E, Güçlü P, Ökten M, Güllü Ü, Şenay Ş, Tektürk MB, Karabulut H, Alhan C. 2012. Is Hepatic and Renal (somatic) OxygenSaturation Followed by NIRS Method during Extracorporeal Circulation in Adult Cardiac Surgery? Journal of Acrbadem University Health Sciences. July Cover 3-Number 3, 164-169. 